\title{
EXIGENCY OF CHANNEL ESTIMATION IN AN OFDM SYSTEM: A SIMULATION BASED STUDY
}

\author{
Ayfer Babaoğlua ${ }^{a *}$, Selva Muratoğlu Çürük ${ }^{b}$ \\ $a^{*}$ Department of Electrical and Electronics Engineering, Faculty of Engineering and Natural Science, \\ Iskenderun Technical University, İskenderun/Hatay, Turkey (corresponding author) \\ ayfer@emtamuhendislik.com.tr \\ ${ }^{b}$ Department of Electrical and Electronics Engineering Faculty of Engineering and Natural Science, \\ Iskenderun Technical University, İskenderun/Hatay, Turkey \\ selva.curuk@iste.edu.tr
}

\begin{abstract}
The popular multicarrier modulation technique, Orthogonal Frequency Division Multiplexing (OFDM), is used for high rate wireless communication systems, as well as for other inexplicit multipath channel applications, such as powerline and underwater communications. Final performance of an OFDM system depends highly on the channel because variations in the characteristics of the channel result with a degradation in the performance. In this study, a modular OFDM system which is modeled using Matlab/Simulink program is given. The proposed model is used for performance evaluation. The variations in the performance of the OFDM system are analyzed by investigating the Bit Error Rate (BER). A comparison is made for two modulation schemes, namely phase-shift keying (M-PSK) and quadrature amplitude modulation (M-QAM), under the additive white Gaussian noise assumption. It has been shown that for a safe communication on a heavy Rayleigh channel especially, channel estimation is a necessity.
\end{abstract}

Keywords: Channel estimation, Matlab, Orthogonal Frequency Division Multiplexing, Simulink.

\section{Introduction}

Orthogonal Frequency Division Multiplexing (OFDM), the well-known widely deployed multicarrier techniques, is a modulation scheme that splits the total transmission bandwidth into a number of subchannels with orthogonal subcarriers in order to cope with multipath channel effects and transmit the symbols. Although OFDM applications had a limited use for a long time due to implementation complexity, with the developments in electronics it has been a popular topic in the field of communications. Nowadays, with its powerful anti-fading capacity and data transfer rate, the OFDM technology is used by many communication 
services in wireless communications as well as in other multipath channel applications, such as powerline and underwater communications and is considered as the core technology of next-generation broadband communications.

The performance of an OFDM system is indeed a well-researched topic and a huge amount of paper related to this scope can be found in the literature. Also there are simulation models; some noteworthy ones are presented in [1-7]. Studies on modelling systems use highly specialized mathematical tools such as Matlab, Simulink [8], Maple, and Mathematica in the literature. These software packages provide built-in libraries and standard building blocks for using in developing of systems. The most popular tool is Matlab/Simulink in communication area which give the advantage of easily modeling channel, modulation, and demodulation with different parameters. For example, in [1] the performance of OFDM is tested for two digital modulation techniques using Matlab/Simulink toolbox. In [2], authors built an OFDM simulation model under environment of Simulink. Similarly in paper [3], a Simulink based simulation system is implemented under Additive White Gaussian Noise channel (AWGN) to study the performance analysis. The authors of [4] have proposed the design of an OFDM system, using Simulink modeling tool and communication block set with PSK digital modulation schemes. Mapping techniques for OFDM are simulated in [5]. The combination of MIMO and OFDM system is given in [6]. The authors of [7] presents a simulation model of OFDM communication system based on again Simulink.

OFDM systems are widely used in different areas under varying channel conditions, with various transceiver designs and different modulation schemes. Thus modular simulation models of specific cases may still be essential for performance analysis. These models provide a platform for further studies of OFDM systems. Further simulation models may also be used for educational demonstration and experimental purposes offering highlighting and studying specific concept and phenomena. In the first step of this study, a simulation model of an OFDM system with Matlab/Simulink is presented. Matlab and Simulink have been chosen as the modeling tool because of their flexibility and mathematical capabilities. Next, the performance of the modeled system is tested under Rayleigh channel and with AWGN assumption. The performance of the OFDM transmitter and receiver is investigated for Rayleigh fading channel and with AWGN; and the variations in performance caused by channel are observed. The results concerning two typical digital modulation schemes of OFDM, namely M-QAM (M-ary Quadrature Amplitude Modulation) and M-PSK (M-ary Phase Shift Keying) are presented. Bit Error Rate (BER) versus Signal to Noise Ratio (SNR) curves are used for comparing the results of M-QAM and M-PSK modulations. It is shown that channel estimation and correction is a critical issue in all OFDM receivers.

The rest of the paper is organized as follows. Section 2 briefly describes the Simulink OFDM system model used in simulations. Simulation results are presented in Section 3. Section 4 follows with conclusions.

\section{OFDM system model}

OFDM is the popular technique for frequency selective multipath channels. An OFDM signal is the sum of $N$ independent QAM/PSK signals modulated onto orthogonal subcarriers. In a frequency selective band, selecting the bandwidth of each subchannel small enough results with flat fading subchannels. Thus, in an OFDM system the bandwidth of each subchannel is small compared to the coherence bandwidth of the channel. That is, the symbol duration is large compared to the channel time dispersion and the subcarriers experience flat fading. 
Figure 1 displays the conventional transceiver of an OFDM system modeled with Matlab / Simulink, which is used in our simulations. The amplitudes and/or phases of the carriers are calculated based on the modulation scheme, namely M-PSK, or M-QAM. Then, a serial-toparallel buffer segments the information sequence into frames, where the bits in the frames are split into $\mathrm{N}$ groups, i.e. each subcarrier is assigned data that will be transmitted. Then an OFDM block follows. Here the spectrum is converted back to its time domain signal using an Inverse Fourier Transform. The Inverse Fast Fourier Transform (IFFT) performs this transformation and provides a simple way of ensuring the orthogonality of subcarriers. The guard period may be added to the beginning of each symbol. The use of a cyclic prefix is also optional, which both preserves the orthogonality of the tones and eliminates intersymbol interference (ISI) between consecutive OFDM symbols.

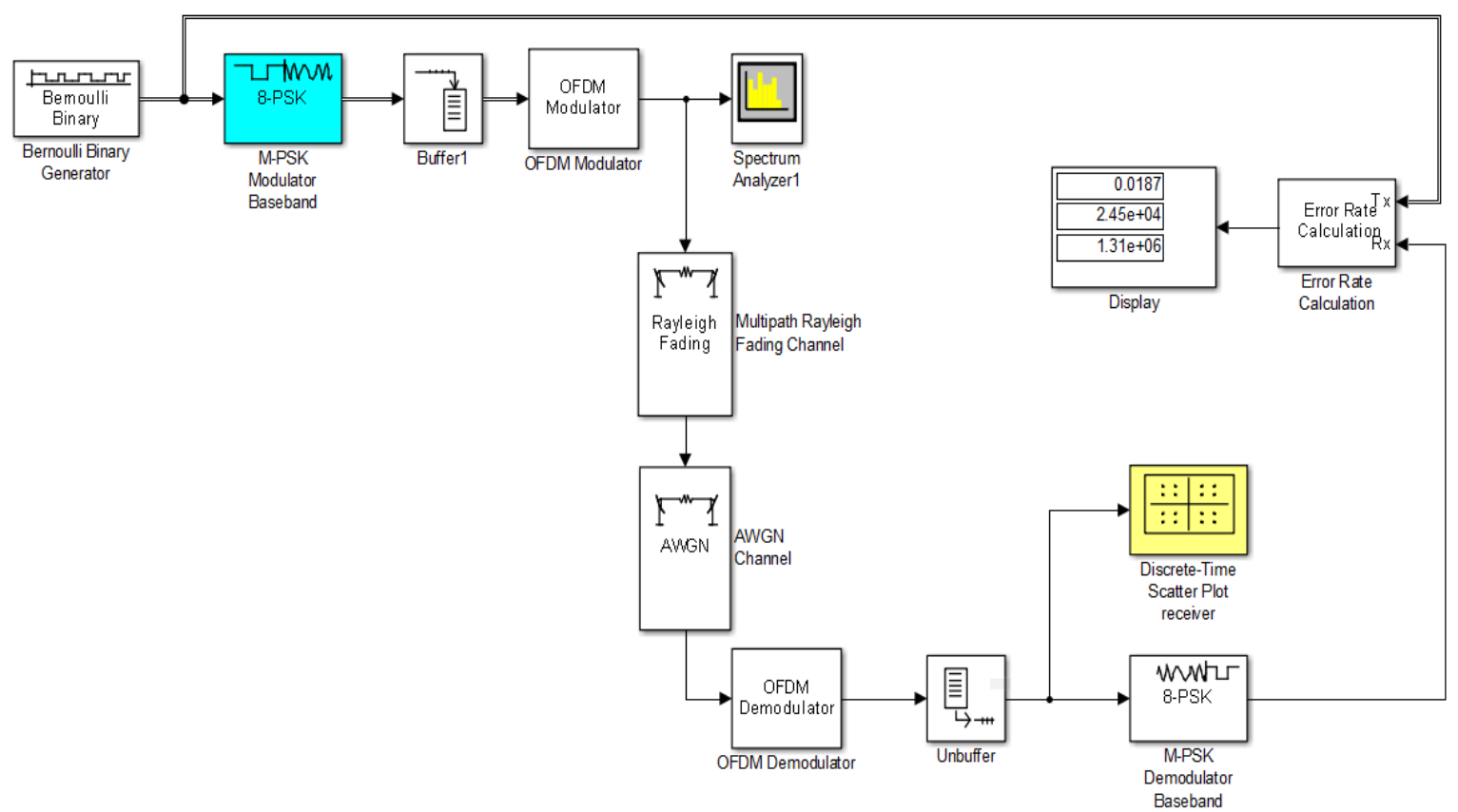

Figure 1. Block diagram of an OFDM system modeled with Matlab/Simulink

The generated signal waveform then passes through the Rayleigh channel with AWGN. This channel parameters can easily be adjusted for a wide variety. The channel output is fed to the receiver. The receiver basically does the reverse operation of the transmitter. First, the guard period and cyclic prefix are removed from the received signal, if they have been added. The FFT of each symbol is then taken to find the original transmitted spectrum. Finally, according to modulation scheme, the phase angle and/or amplitude of each carrier is evaluated and converted back to the data word. Finally the data words are then combined to obtain the original transmitted data. The performance is analyzed by comparing the transmitted and received data and calculating BERs.

\section{Simulation results}

In this section, we analyze the performance of the OFDM simulator explained in the previous section under the assumption of AWGN. The number of subcarriers in each OFDM symbol is selected to be 128, and frames are sent through the channel with modulation M-PSK and MQAM. The BER results are calculated after 1000000 bits. 
First we compare the performance of OFDM system with PSK and QAM modulation schemes with various modulation levels. For this part, we assume that channel effects are removed completely and we analyze the performance of modulation with different levels only. Figure 2 and Figure 3 give BER versus SNR graphs for PSK modulation with $\mathrm{M}=2,4$ and 8 levels and QAM modulation with $\mathrm{M}=4,8$ and 16 levels for $0-30 \mathrm{~dB}$ SNR range, respectively. As expected, for both modulation schemes as SNR increases the performance gets better. Also as the level increases the BER increases, since the signals in the cancellation diagram get closer.

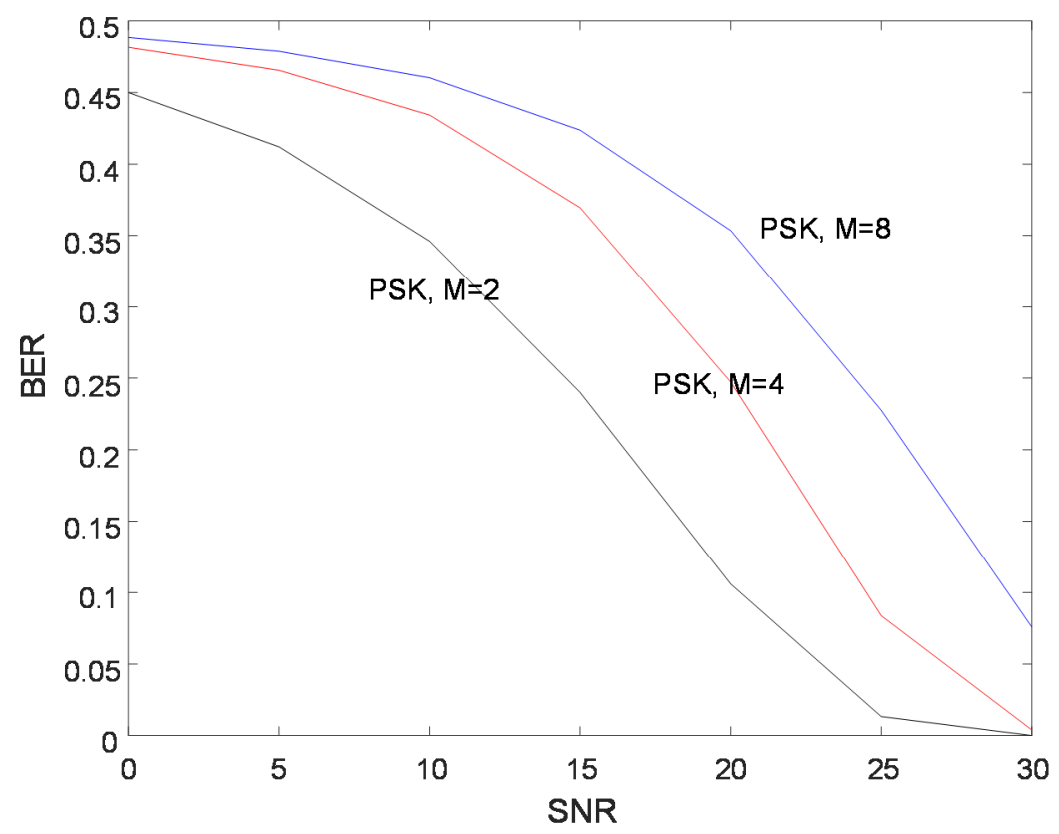

Figure 2. BER vs SNR graph, PSK modulation, $M=2,4,8$

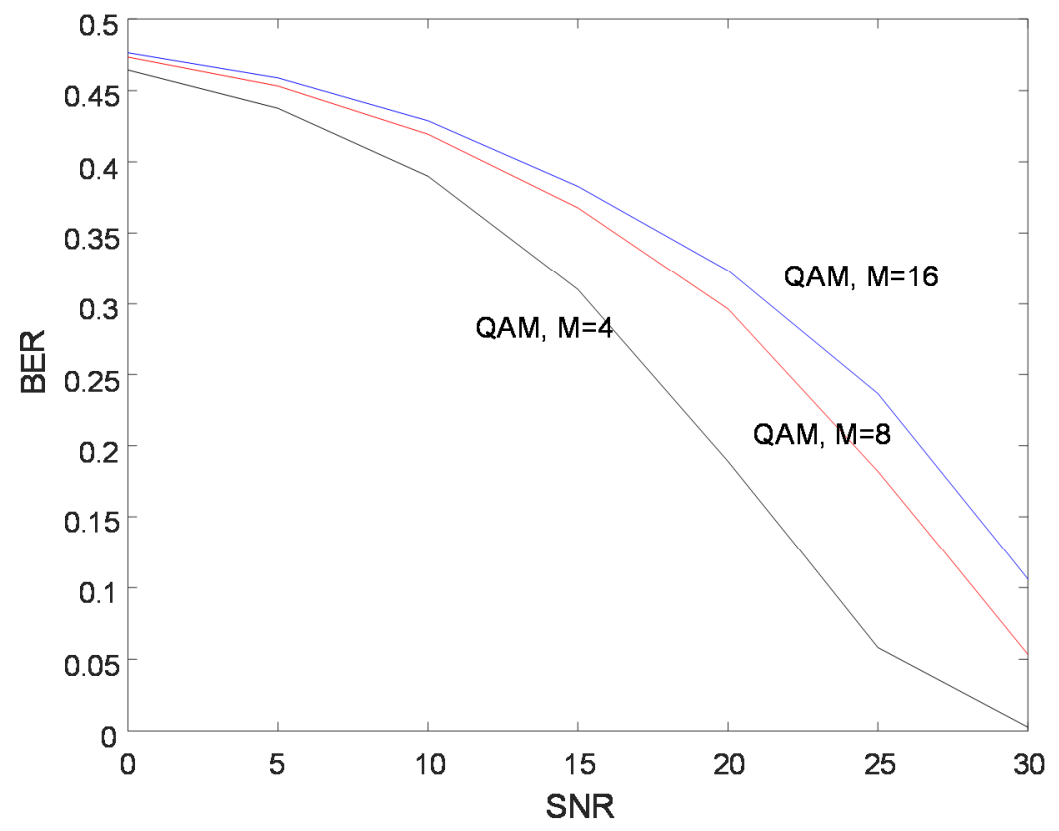

Figure 3. BER vs SNR graph, QAM modulation, $M=4,8,16$ 
In an OFDM system, the generated waveform is said to be disturbed during the transmission through the channel, since the channel introduces amplitude and phase shifts due to frequency selective and time varying nature. The question is that if the receiver needs to take into account these unknown changes to acquire the original bits or not. Therefore we investigate the performance of the OFDM system under the Rayleigh fading channel and AWGN assumption. The transmitted signals are 4-PSK modulated. Two multipath Rayleigh fading channels are generated for the simulation. The first one is modeled as a two tapped delay line, with path delays $\left[\begin{array}{ll}0 & 10^{-4}\end{array}\right]$ and path gains $\left[\begin{array}{ll}0 & 0.4\end{array}\right]$. The second channel is a five tapped

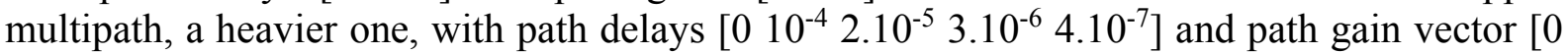

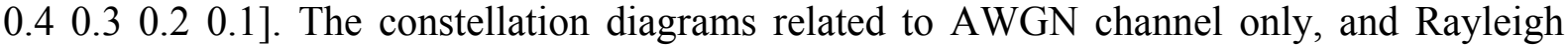
channels with 2-tapped and 5 tapped multipath are given in Figure 4, Figure 5 and Figure 6, respectively. All the plots are given for $\mathrm{SNR}=30 \mathrm{~dB}$ and maximum Doppler shift is selected to be very low $\left(10^{-8} \mathrm{~Hz}\right)$ in order to see the effect of multipath only. It is easily seen from the figures that the performance loss is intolerable even for slight multipath case. (The BER performance is expected to be very bad compared to AWGN channel.) Thus the OFDM receiver needs estimation and equalizing, i.e, the channel factors should be estimated and compensated prior the detector in the case of multipath.

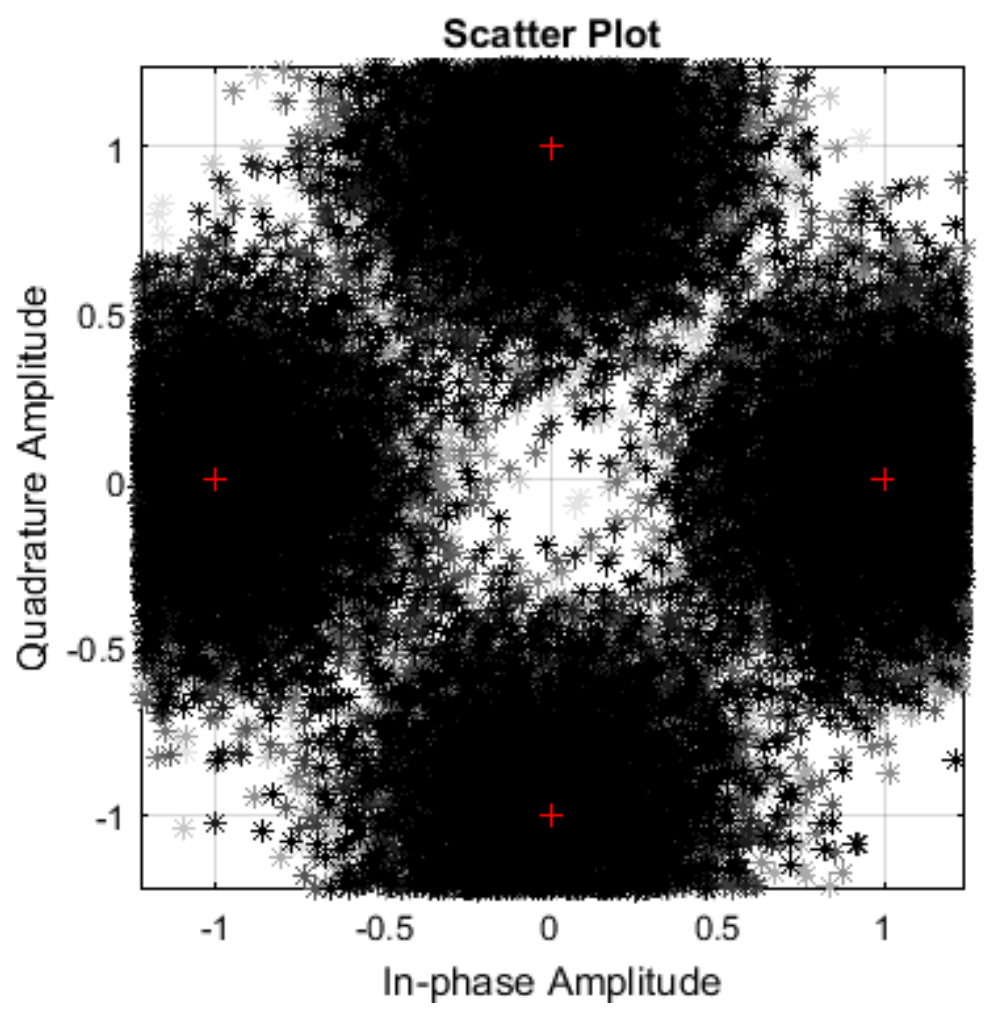

Figure 4. Constellation diagram, 4-PSK, AWGN channel, $S N R=30 \mathrm{~dB}$. 


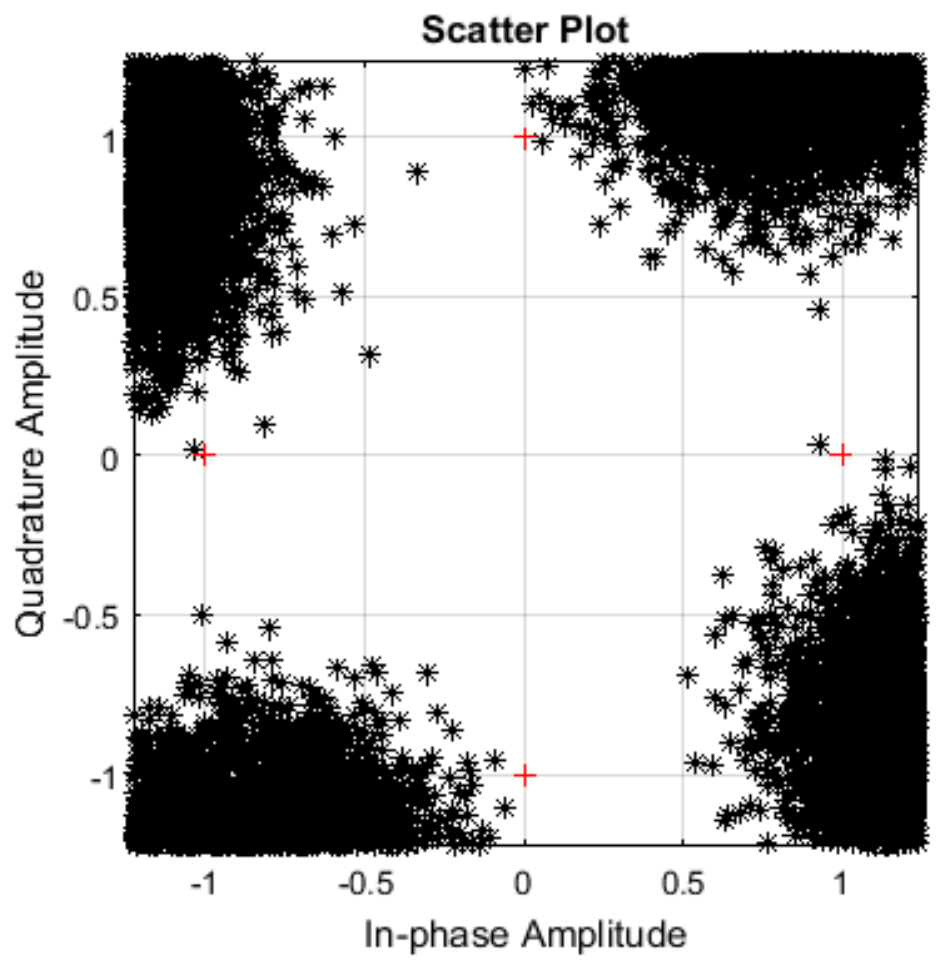

Figure 5. Constellation diagram, 4-PSK, 2-tapped delay channel with $A W G N, S N R=30 \mathrm{~dB}$.

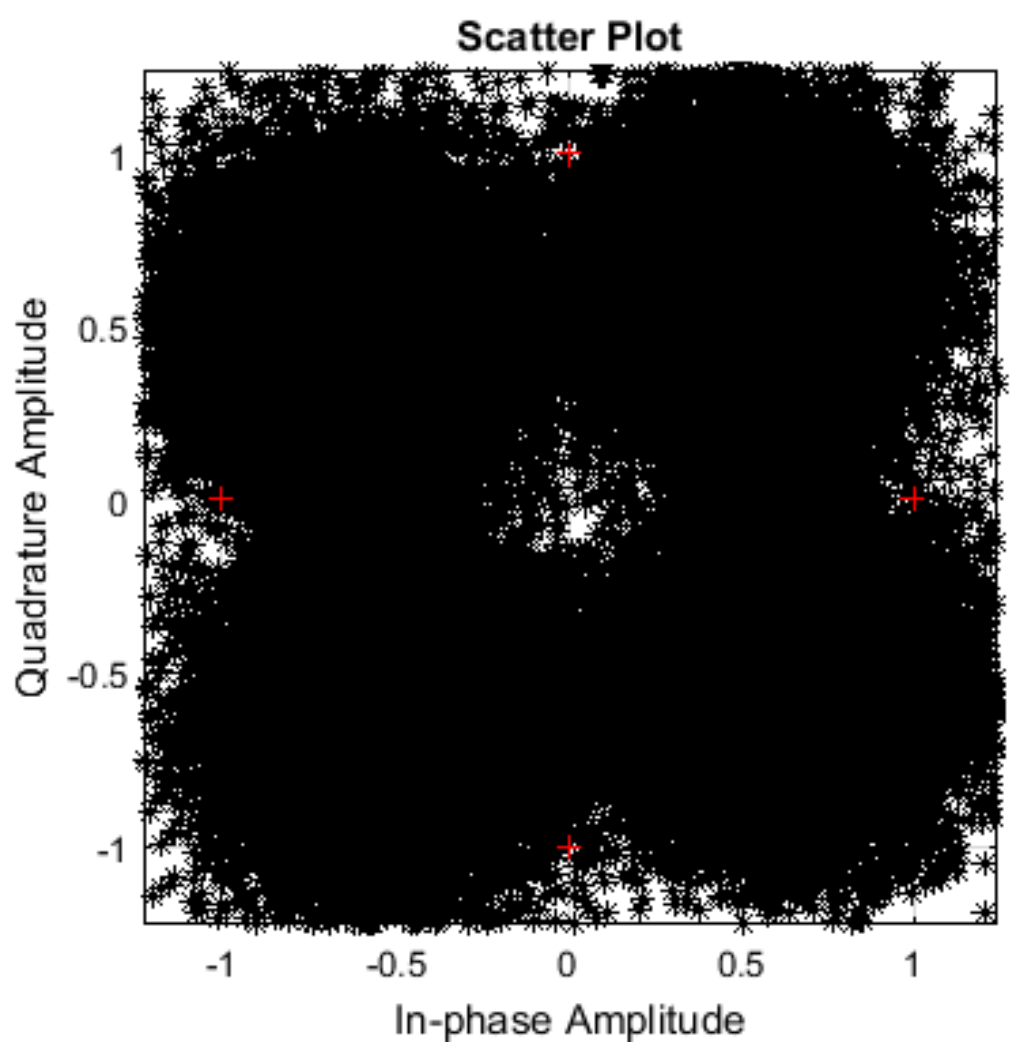

Figure 6. Constellation diagram, 4-PSK, 5-tapped delay channel with $A W G N, S N R=30 \mathrm{~dB}$. 
The estimation and equalization methods that can be used depend on the channel and the desired complexity of the receiver. There are two basic channel estimation methods in the literature: The Least Square (LS) algorithm and the Linear Minimum Mean Square Error (LMMSE) algorithm [9, 10]. The goal of LS estimator is to minimize the square distance between the received and the original signal. The LS estimation method is simple, but since it does not use the related features of the channel, the accuracy of the estimation is low. The LMMSE estimator minimizes the estimation mean square errors and has a better accuracy. This algorithm needs additional information, such as SNR and channel characteristics and has a higher complexity. Although the LMMSE estimator is more complex, we suggest using it because of its performance especially for heavy multipath channel conditions.

\section{Conclusions}

We built an OFDM simulation model using interactive environment of Matlab/Simulink tool which gives opportunity for studying the OFDM system and it can be enlarged easily because of the modular structure. Using this model, the performance of OFDM system is analyzed for two typical digital modulation techniques, namely M-PSK and M-QAM. The results obtained for BER versus SNR graphs show low BER values for larger value of SNR, as expected. It is also observed that although high value of $\mathrm{M}$ increases spectrum efficiency, this modulation schemes are easily affected by noise. Thus, OFDM systems with higher M-ary modulation schemes may be used for large capacity applications but with the cost of an increase in BER. We have also considered the performance of the OFDM system under Rayleigh fading channel. Simulation results show that the receiver is in need of channel estimation and equalizing blocks, with a cost of the complexity, for a satisfactory performance. In the Simulink model proposed, according to actual needs, a suitable channel estimation algorithm can be added. Although it will increase the complexity, preferring LMMSE estimator is advised because there is a great degradation in the performance of the receiver especially under 5-tap Rayleigh channel and it will provide better performance.

\section{References}

[1] Patel, Jigisha N. and Upena D. Dalal. "A Comparative Performance Analysis of OFDM using MATLAB Simulation with M-PSK and M-QAM Mapping," International Conference on Computational Intelligence and Multimedia Applications, Sivakasi, Tamil Nadu, India, December 13-15, 2007.

[2] Zhang, Jun and Zhongpei Zhang, "Simulation and Analysis of OFDM System Based on Simulink," International Conference on Communications, Circuits and Systems, International Conference Center, UESTC, Chengdu, China, July 28-30, 2010.

[3] T. P., Surekha, T. Ananthapadmanabha and C. Puttamadappa, "Modeling and Performance Analysis of QAM-OFDM System with AWGN Channel", Third PacificAsia Conference on Circuits, Communications and System, Wuhan, China, July 17-18, 2011.

[4] Pokle, S. B. and K. D. Kulat Fiete, "MATLAB Simulation of a Wireless Communication System using OFDM Principle”, IETE Technical Review, 23:3 (2006), 187-198. (Published online: 26 Nov 2015)

[5] Mahor, Devesh and Kamlesh Kumar Yadav, "Investigating Mapping Technique Over Statistical Parameter under Noisy Channel for Wireless Communication", 5th 
International Conference on Confluence - The Next Generation Information Technology Summit, Noida, India, Sept.25-26, 2014.

[6] Ogale, Archana, Shubhangi Chaoudhary and A. J. Patil, "Performance Evaluation of MIMO-OFDM System using Matlab / Simulink With Real Time Image Input”, 10th IEEE and IFIP International Conference on Wireless and Optical Communications Networks, Bhopal, India, July 26-28, 2013.

[7] Qiang Li, Tong and Li Gang Xu, "Study and Simulation Analysis of OFDM System Based on Simulink", 3rd International Conference on Mechatronics and Intelligent Materials: Advanced Materials Research, 706:708 (2013) 1967-1970.

[8] http://www.mathworks.com/help/simulink/, The Matlab Simulink website.

[9] Khlifi, Abdelhakim and Ridha Bouallegue, "Performance Analysis of LS and LMMSE Channel Estimation Techniques for LTE Downlink Systems", International Journal of Wireless \& Mobile Networks: Networking and Internet Architecture, 3: 5 (2011), 141149. (Published online: 7 Nov 2011)

[10] Zettas, Spiridon, Pavlos. I. Lazaridis and Zaharias D. Zaharis, et al. "Performance Comparison of LS, LMMSE and Adaptive Averaging Channel Estimation (AACE) for DVB-T2", 10th IEEE International Symposium on Broadband Multimedia Systems and Broadcasting, Ghent, Belgium, June 17-19, 2015. 